Keywords: alpha-enolase; Notch1; biomarker; chemoresistance; cisplatin; head and neck squamous cell carcinoma; iTRAQ; tandem mass spectrometry

\title{
Identification of chemoresistant factors by protein expression analysis with iTRAQ for head and neck carcinoma
}

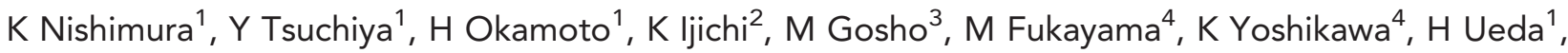 \\ C R Bradford ${ }^{5}$, T E Carey ${ }^{5}$ and T Ogawa ${ }^{*}, 1$
}

${ }^{1}$ Department of Otorhinolaryngology, Aichi Medical University School of Medicine, 1-1 Yazakokarimata, Nagakute, Aichi 480-1195, Japan; ${ }^{2}$ Department of Otolaryngology, Head and Neck Surgery, Nagoya City University Graduate School of Medical Science, 1-1 Yazakokarimata, Nagakute, Aichi 480-1195, Japan; ${ }^{3}$ Unit of Biostatistics, Advanced Medical Research Center, Aichi Medical University School of Medicine, 1-1 Yazakokarimata, Nagakute, Aichi 480-1195, Japan; ${ }^{4}$ Division of Clinical Research Promotion, Institute of Comprehensive Medical Research, Aichi Medical University School of Medicine, 1-1 Yazakokarimata, Nagakute, Aichi 480-1195, Japan and ${ }^{5}$ Department of Otolaryngology-Head and Neck Surgery, University of Michigan, Taubman Center Floor 1 Reception A, 1500 E Medical Center Drive SPC 5312, Ann Arbor, MI 48109, USA

Background: Cisplatin and other anticancer drugs are important in the treatment of head and neck squamous cell carcinoma; however, some tumours develop drug resistance. If chemoresistance could be determined before treatment, unnecessary drug administration would be avoided. Here, we investigated chemoresistance factors by comprehensive analyses at the protein level.

Methods: Four human carcinoma cell lines were used: cisplatin-sensitive UM-SCC-23, UM-SCC-23-CDDPR with acquired cisplatin resistance, naturally cisplatin-resistant UM-SCC-81B, and UM-SCC-23/WR with acquired 5-fluorouracil resistance. Extracted proteins were labelled with ITRAQ and analysed by tandem mass spectrometry to identify resistance. Protein expression was confirmed by western blotting and functional analysis was carried out using siRNA.

Results: Thirteen multiple-drug resistance proteins were identified, as well as seven proteins with specific resistance to cisplatin, including $\alpha$-enolase. Differential expression of these proteins in cisplatin-resistant and -sensitive cell lines was confirmed by western blotting. Functional analysis for $\alpha$-enolase by siRNA showed that cisplatin sensitivity significantly was increased in UM-SCC-81B and slightly in UM-SCC-23-CDDPR but not in UM-SCC-23/WR cells.

Conclusions: We identified proteins thought to mediate anticancer drug resistance using recent proteome technology and identified $\alpha$-enolase as a true cisplatin chemoresistance factor. Such proteins could be used as biomarkers for anticancer agent resistance and as targets of cancer therapy.

Head and neck squamous cell carcinoma (HNSCC) is the sixth most common cancer worldwide, constituting around $5 \%$ of the total number of cases. Annually, 500000 new cases are reported worldwide (Boring et al, 1993). Recently, improvements in surgery, radiotherapy, and chemotherapy have contributed to greater preservation of organs and better quality of life for affected patients, but the survival rate has not improved (Vokes et al, 1993; Mignogna et al, 2004).

Anticancer drugs have a major role in functional preservation in HNSCC. At present, the key anticancer drug for advanced HNSCC is still cisplatin (cis-diamminedichloroplatinum; CDDP), although not all cases have a good response to the drug and some tumours

*Correspondence: Professor T Ogawa; E-mail: ogawate@aichi-med-u.ac.jp

Received 9 December 2013; revised 13 June 2014; accepted 20 June 2014; published online 17 July 2014

(c) 2014 Cancer Research UK. All rights reserved 0007-0920/14 
show CDDP resistance. In addition to the cost of the drug, CDDP administration in such resistant cases would provide little curative effect but could increase the chances of adverse drug reactions, which is a clear disadvantage. It would be of great clinical importance if tumour sensitivity to the drug could be predicted before its administration, as it would prevent needless dosage. Consequently, the identification of CDDP-resistant mechanisms has been attempted by various techniques such as transcriptomics. However, as this assesses mRNA expression levels, the method does not enable detection of the true chemoresistant factors because it does not explore protein-dependent mechanisms (Chung et al, 2004, 2006).

Recently, through the development of mass spectrometry (MS), analysis of the whole protein by proteomics has been applied in cancer research (Chung et al, 2007; He et al, 2009; Craft et al, 2013). For example, a recent useful tool for proteomics is peptide mass fingerprinting (PMF), which is performed on proteins isolated by two-dimensional electrophoresis to identify candidate proteins discovered during production. However, studies based on only these methods of MS are not reproducible. Therefore, in the present study, we attempted to detect the true chemoresistant factors of HNSCC by performing a comprehensive analysis of CDDP resistance at the protein level using the isobaric tags for Relative and Absolute Quantification (iTRAQ) method, which is a new technology involving stable isotopes, nano-high-performance liquid chromatography, and tandem mass spectrometry (MS/MS). In addition, to exclude proteins related to multidrug resistance (MDR) and to identify only true CDDP-resistant factors, we carried out our analyses simultaneously in a fluorouracil (5-FU)-resistant cell line. Previous methods would not have enabled these comparisons to be made or for several samples to be identified at the same time, but using the iTRAQ method this was made possible. We also performed western blotting and siRNA analysis to determine protein expression and to perform functional analysis of the candidate protein involved in CDDP resistance. Also now Notch1 have been becoming important molecules for head and neck squamous cell carcinoma (Pickering et al, 2013); we also checked these expressions on them.

\section{MATERIALS AND METHODS}

Cell lines. The HNSCC cell lines UM-SCC-23 and UM-SCC-81B were a gift from Dr Thomas E. Carey, University of Michigan (Ann Arbor, MI, USA) (Brenner et al, 2010). The cell line UM-SCC-23CDDPR with acquired CDDP resistance was established by repeated CDDP exposure. For the 5-FU acquired resistant cell line, UM-SCC-23/WR was kindly provided by Dr Kei Ijichi, Nagoya City University (Ijichi et al, 2008; Murata et al, 2011). Cells were cultured in RPMI 1640 medium with $10 \%$ fetal bovine serum (IBL, Tokyo, Japan), $100 \mathrm{U} \mathrm{ml}^{-1}$ penicillin, and $0.1 \mathrm{mg} \mathrm{ml}^{-1}$ streptomycin (SIGMA, St Louis, MO, USA) at $37^{\circ} \mathrm{C}$ and $5 \% \mathrm{CO}_{2}$. The doubling time was the same in four cell line.

Cell survival assay. Cell lines were cultured with CDDP (Nippon Kayaku, Tokyo, Japan) at various concentrations $(0,1.56,3.12$, $6.25,12.5$, and $25 \mu \mathrm{g} \mathrm{ml}^{-1}$ ) in 96-well flat-bottom plates (Falcon 3072; Becton Dickinson, Franklin Lakes, NJ, USA). The effect of CDDP on cell growth and division was determined by comparing the cell number at the start of the culture with that 3 days later using the WST-1 assay kit (Cell Counting Kit; DOJINDO, Tokyo, Japan). WST-1 reagent was added $10 \mu \mathrm{l}$ per well, cultured for $3 \mathrm{~h}$, and the coloration value was measured using a 96-well plate reader (dual wavelength: $450 \mathrm{~nm}$ and $640 \mathrm{~nm}$; Spectramax M5; Molecular Devices, Sunnyvale, CA, USA).
Protein sample preparation and iTRAQ labelling. CDDPsensitive, acquired resistant, naturally resistant, and 5-FU acquired resistant cell lines were grown to $80 \%$ confluency in Falcon T-25 flasks (Becton Dickinson). Cells were washed with PBS and collected by incubation with $2 \mathrm{ml}$ cellular lysis buffer ( $8 \mathrm{M}$ urea/2\% CHAPS/1 mM DTT). Ultrasonic processing ( $50 \mathrm{~W}$ for $30 \mathrm{~s}$ ) of this solution was then carried out using an ultrasonic processor (Sonifier cell disruptor; Heat Systems-Ultrasonics Inc., Plainview, LI, USA). After centrifugation at $10000 \mathrm{~g}$ for $10 \mathrm{~min}$, the cells were lysed and passed through a $0.45 \mu \mathrm{m}$ filter to remove insoluble matter. Samples were stored at $-80{ }^{\circ} \mathrm{C}$ until required.

The protein density of the cellular lysis buffer was determined to enable concentrations to be adjusted to identical levels. From each sample, $100 \mathrm{mg}$ of proteins was mixed with acetone, precipitated overnight at $-20^{\circ} \mathrm{C}$, dissolved in lysis buffer, denatured, and the cysteines were blocked as described in the iTRAQ protocol (Applied Biosystems, Framingham, MA, USA). Each sample was digested with $20 \mathrm{ml} 0.1 \mathrm{mg} \mathrm{ml}^{-1}$ trypsin solution (AB Sciex, Ontario, Canada) at $37^{\circ} \mathrm{C}$ overnight and labelled with the iTRAQ tags as follows: (i) the cisplatin-sensitive cell line UM-SCC-23 with the $114 \mathrm{tag}$, (ii) the cisplatin-acquired resistant cell line UM-SCC23-CDDPR with the 115 tag, (iii) the cisplatin-naturally resistant cell line UM-SCC-81B with the 116 tag, and (iv) the 5-FU acquired resistant cell line UM-SCC/WR with the 117 tag (Figure 1).

Liquid chromatography tandem mass spectrometry (LC-MS/MS) analysis. To reduce sample complexity during LC-MS/MS analysis, the pooled samples were diluted 10-fold with SCX buffer (ICAT Cation Exchange Buffer Pack) and run through a $2.1 \times 200 \mathrm{~mm}$ polysulfoethyl A SCX column (AB Sciex). Nanoflow electrospray ionisation MS/MS analysis of peptide samples was carried out using the Triple TOF5600 system (AB Sciex) interfaced with the Dina-A nanoflow LC system (KYA, Tokyo, Japan). The chromatographic capillary columns were packed with Sep-Pak (Waters Co., Milford, MA, USA) and reversed phase material in 100\% acetonitrile (ACN) at a pressure of 1000 p.s.i. The peptide sample from each SCX fraction was enriched using a trap column (HiQ sil C18W-3; KYA) at a flow rate of $3 \mu \mathrm{min}^{-1}$ and separated on an analytical column (HiQ sil C18W-3: $100 \mu \mathrm{m} \times 100 \mathrm{~mm}$; KYA) at a flow rate of $300 \mathrm{nl} \mathrm{min}{ }^{-1}$. The peptides were eluted using a linear gradient of 2$80 \%$ ACN over $180 \mathrm{~min}$. MS analysis was carried out in a datadependent manner with full scans acquired using the Triple TOF mass analyser at a mass resolution of 60000 at $400 \mathrm{~m} / z$. For each MS cycle, the 10 highest intense precursor ions from a survey scan were selected for MS/MS.

Protein identification and quantification. MS and MS/MS data searches were carried out using ProteinPilot software (4.0.8085; AB Sciex) based on the workflow with a spectrum selector and reporter ion quantifier. Searches used a Sequest search algorithm and the UniProt, Swiss-Prot human RefSeq databases. Precursor and fragment mass tolerance were set to 20 p.p.m. and $0.1 \mathrm{Da}$, respectively. Peptide and protein data were extracted using high peptide confidence and top one peptide rank filters. The false discovery rate (FDR) was calculated by enabling the peptide sequence analysis using a decoy database. High confidence peptide identifications were obtained by setting a target FDR threshold of $1 \%$ at the peptide level.

Western blotting analysis. Cells were washed with PBS, then collected by incubation with $2 \mathrm{ml}$ cellular lysis buffer. Ultrasonic processing ( $50 \mathrm{~W}$ for $30 \mathrm{~s}$ ) of this solution was then carried out using an ultrasonic processor (Sonifier cell disruptor; Heat Systems-Ultrasonics Inc.). After centrifugation at $10000 \mathrm{~g}$ for $10 \mathrm{~min}$, the cells were lysed and passed through a $0.45 \mu \mathrm{m}$ filter to remove insoluble matter. Samples were stored at $-80^{\circ} \mathrm{C}$ until required. 


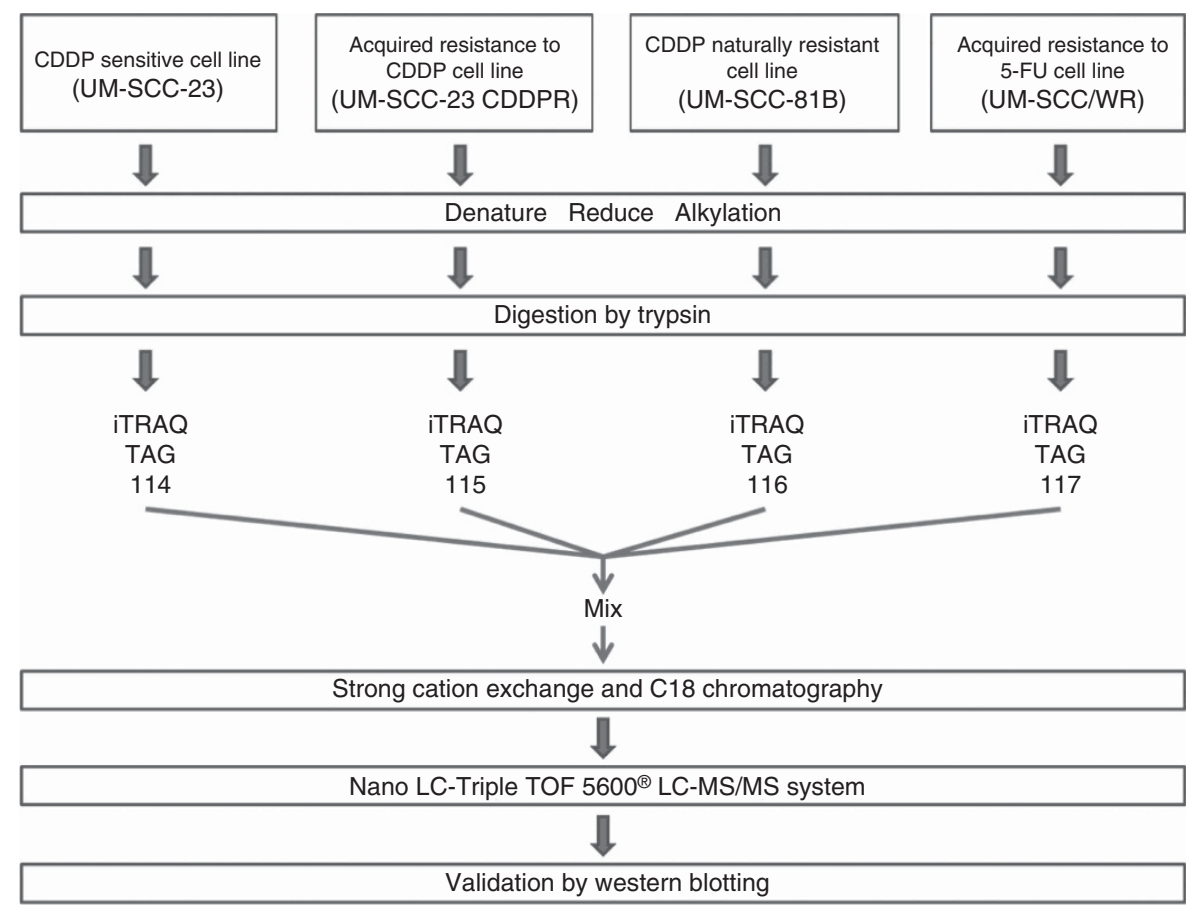

Figure 1. Flowchart of iTRAQ proteomics approach. Samples were treated according to the iTRAQ protocol, including the blocking of cysteines. They were then labelled with ITRAQ tags and analysed by the nano-LC and Triple TOF5600 system. Protein expression was confirmed by western blotting.

Extracted proteins were run on $12.5-15 \%$ polyacrylamide gels (SuperSepAce; Wako, Osaka, Japan) and blotted on polyvinylidene difluoride membranes (Immobilon-P; Millipore, Billerica, MA, USA). Membranes were incubated with 5\% skim milk and $1 \%$ normal goat serum in PBS or TBS to prevent nonspecific binding, then reacted with the primary antibody. Primary antibodies used were anti-X-ray repair cross-complementing protein 1 (XRCC1), anti-calreticlin, anti-glutathione S-transferase (GST), anti- $\alpha$-enolase (all Abcam, Cambridge, UK), and anti-Notch1 (Cell Signaling Technology, Danvers, MA, USA). Secondary antibodies were Envision + System- HRP Labeled Polymer Anti-Rabbit, and Envision + System- HRP Labeled Polymer Anti-Mouse (Dako North America, Inc., Carpinteria, CA, USA). After incubation with secondary antibodies, the ECL plus Western Blotting Detection system (GE Healthcare UK Ltd, Little Chalfont, UK) was used to detect bands with a luminous detector (LAS 1000 lite and LAS 4000; GE Healthcare UK Ltd).

Transfection of siRNAs. siRNAs against $\alpha$-enolase were purchased from Invitrogen (ENO1HSS103243; Carlsbad, CA, USA) with sequence $5^{\prime}$-UUCGAUAGACACCACUGGGUAGUCC- $3^{\prime}$. Cells were plated in 24-well plates and transfected with $6 \mathrm{pmol}$ si $\alpha$-enolase or Stealth RNAi Negative Control high GC Duplex using Lipofectamine 2000 (Invitrogen). The next day, cells were re-plated in 96-well plates and allowed to attach overnight. Cells were then incubated with or without each drug for an additional $96 \mathrm{~h}$, and cell growth was measured using the WST-1 assay (Cell Counting Kit; DOJINDO).

Statistical analysis. The half maximal inhibitory concentration (IC50) was used to evaluate the relationship between the CDDP or 5 -FU concentration and cell survival response. The IC50 was estimated by using a nonlinear mixed effect model. The cell survival response was modelled by $100 /\{1+$ (concentration/ IC50 $\left.)^{\gamma}\right\}$, where IC50 $=\exp \left(\beta_{0}+\beta_{1} \mathrm{D}+\right.$ between-subject $)$ and hillcoefficient $\gamma=\exp \left(\beta_{2}+\beta_{3} \mathrm{D}\right)$. Here, $\beta_{0}-\beta_{3}$ are fixed effects, the between-subject is a random effect, and $\mathrm{D}=1$ (with $\alpha$-enolase), 0 (without $\alpha$-enolase) is the dummy variable. In particular, $\beta_{1}$ is corresponding to the difference in log-transformed IC50 between with and without $\alpha$-enolase. Based on the estimation of $\beta_{1}$ in the above model, the IC50 was compared between the two treatments (with and without si $\alpha$-enolase) using the ratio of IC50 and Student's $t$-test for the null hypothesis of zero difference in $\log$-transformed IC50 (that is, testing for $\beta_{1}=0$ ). All tests were two-sided and the significance level was set at 0.05. Statistical analyses were carried out using SAS 9.3 software (SAS institute Inc., Cary, NC, USA).

\section{RESULTS}

Sensitivity test of cell lines for CDDP and 5-FU. We investigated the sensitivity of the UM-SCC-23, UM-SCC-23-CDDPR, and UMSCC-81B cell lines to CDDP using the WST-1 assay. As shown in Figure 2, UM-SCC-23 was the most CDDP-sensitive cell line of the three. This result indicates that UM-SCC-23-CDDPR possesses acquired CDDP resistance and that UM-SCC-81B is naturally CDDP resistant. For the 5-FU acquired resistant cell line, UM-SCC-23/WR was 1.4 times more resistant to 5-FU than was UM-SCC-23 (Murata et al, 2011).

Identification and comparison of CDDP-resistant proteins using iTRAQ. We identified a total of 2173 proteins by comparing expression in the different cell lines using iTRAQ. The reproducibly rate, or critical FDR, was $1.0 \%$. Therefore, these proteins appeared to be highly sensitive marker proteins for HNSCC cell lines. Statistical calculations for iTRAQ-based detection and relative quantification were then made by applying the Paragon Algorithm using ProteinPilot Software (AB Sciex). A total of 13 proteins expressed at lower levels in UM-SCC-23 (CDDP-sensitive) cells but at increased levels in UM-SCC-23CDDPR and UM-SCC-81B (CDDP-resistant) cells and in UM-SCC-23/WR (5-FU-resistant) cells were placed in the MDR (multidrug resistance) group $(P<0.05)$. Those proteins with decreased expression in UM-SCC-23 (CDDP-sensitive) and 
UM-SCC-23/WR (5-FU-resistant) cells but increased expression in UM-SCC-23-CDDPR and UM-SCC-81B (CDDP-resistant) cells were regarded as CDDP-specific resistant. A total of seven proteins were placed in this group $(P<0.05$; Table 1$)$. Representative MS/MS spectra of select peptides with their reporter ions are shown for the four proteins XRCC1, calreticulin, GST, and $\alpha$-enolase (Figure 3).

Verification by western blotting. We performed western blotting analysis to confirm the accuracy of iTRAQ in identifying XRCC1 and calreticulin as MDR proteins, and GST and $\alpha$-enolase as CDDP-resistant proteins. Figure 4 shows that expression patterns were identical between the two analyses. As the figure shows, each expression pattern of the proteins corresponds to the iTRAQ results for MDR and for CDDP-resistant proteins. The expression

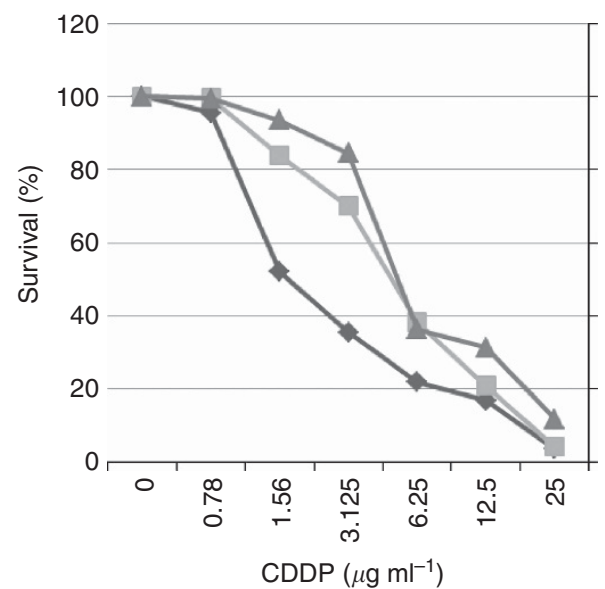

Figure 2. Characterisation of cell lines. Assessment of cell survival following treatment with different concentrations of CDDP according to the WST-1 assay. Black diamonds represent UM-SCC-23 cells, light grey squares represent UM-SCC-23-CDDPR cells, and dark gray triangles represent UM-SCC-81B cells.

Table 1. Differentially expressed proteins

\begin{tabular}{|c|c|c|c|}
\hline Accession No. & Protein name & $\begin{array}{c}\% \\
\text { Coverage }\end{array}$ & $N$ \\
\hline \multicolumn{4}{|l|}{ MDR proteins } \\
\hline $\begin{array}{l}\text { sp|P10809 } \\
\text { sp|P05787 } \\
\text { sp|P13647| } \\
\text { cont|000137 } \\
\text { sp|P14618| } \\
\text { sp|P00338| } \\
\text { sp|P27797 } \\
\text { sp|Q04695| } \\
\text { sp|P12956 } \\
\text { sp|P04264| } \\
\text { sp|P80723 } \\
\text { sp|Q8|Y81| } \\
\text { sp|Q09666| }\end{array}$ & $\begin{array}{l}60 \text { kDa heat shock protein } \\
\text { Keratin, type II cytoskeletal } 8 \\
\text { Keratin, type II cytoskeletal } 5 \\
\text { Cationic trypsin precursor } \\
\text { Pyruvate kinase isozymes M1/M2 } \\
\text { L-lactate dehydrogenase A chain } \\
\text { Calreticulin } \\
\text { Keratin, type I cytoskeletal } 17 \\
\text { X-ray repair cross-complementing protein } 1 \\
\text { Keratin, type II cytoskeletal } 1 \\
\text { Brain acid soluble protein } 1 \\
\text { Putative rRNA methyltransferase } 3 \\
\text { Neuroblast differentiation-associated protein }\end{array}$ & $\begin{array}{l}83.6 \\
86.1 \\
62.4 \\
91.8 \\
77.4 \\
80.4 \\
77 \\
60 \\
34.2 \\
32.8 \\
51.1 \\
16.1 \\
20.9\end{array}$ & $\begin{array}{r}10 \\
11 \\
17 \\
20 \\
23 \\
46 \\
59 \\
77 \\
84 \\
181 \\
449 \\
344 \\
2\end{array}$ \\
\hline \multicolumn{4}{|c|}{ CDDP-specific resistance proteins } \\
\hline $\begin{array}{l}\text { sp|P35579 } \\
\text { sp|P06733 } \\
\text { sp|P02538 } \\
\text { sp|P09211 } \\
\text { sp|Q9NZM1 } \\
\text { sp|P29034 } \\
\text { sp|P23526 }\end{array}$ & $\begin{array}{l}\text { Myosin-9 } \\
\alpha \text {-Enolase } \\
\text { Keratin, type II cytoskeletal 6A } \\
\text { Glutathione S-transferase P } \\
\text { Myoferlin } \\
\text { Protein S100-A2 } \\
\text { Adenosylhomocysteinase }\end{array}$ & $\begin{array}{l}32.7 \\
77.7 \\
74.4 \\
70 \\
54 \\
56.1 \\
39.4 \\
\end{array}$ & $\begin{array}{r}1 \\
24 \\
54 \\
97 \\
80 \\
298 \\
142 \\
\end{array}$ \\
\hline
\end{tabular}

of Notch1 was seen in all the cell lines but slightly decreased in UM-SCC-81B (Figure 5).

Functional analysis using siRNA knockdown. Functional analysis using siRNA was performed of the putative CDDP-resistant protein $\alpha$-enolase. The $\alpha$-enolase knockdown effect was analysed by western blotting in UM-SCC-23 (CDDP-sensitive) and UMSCC-23-CDDPR (CDDP-resistant) cell lines (Figure 6). As this figure shows, siRNA knockdown was successful in the UM-SCC-23 and UM-SCC-23-CDDPR cell lines. Moreover, the effect of a combination of $\alpha$-enolase siRNA and 5 -FU on the proliferation of UM-SCC-23/WR (5-FU-resistant) cells was examined by the WST1 assay. Figure 7A shows only a slight difference between the survival cell response of UM-SCC-23/WR cells to 5-FU alone and to 5 -FU with $\alpha$-enolase siRNA. In addition, the IC50 for 5 -FU in the cell line was not significantly different between the two treatments $(P=0.10)$, as shown in Figure 7D. Figure 7B shows a slight difference between the survival cell response of UM-SCC-23CDDPR cells by CDDP alone and by CDDP with $\alpha$-enolase siRNA. The IC50 for CDDP in the cell line was not significantly different between the two treatments $(P=0.09)$, as shown in Figure 7D.

However, the effect of combination treatment using $\alpha$-enolase siRNA and CDDP on the proliferation of UM-SCC-81B (naturally CDDP-resistant) cells was significant (Figure 7C). The IC50 of CDDP with $\alpha$-enolase was lower than that for CDDP alone (CDDP: 5.38; CDDP + si $\alpha$-enolase: $2.34 ; P<0.001$ ) (Figure $7 \mathrm{C}$ and $\mathrm{D}$ ).

\section{DISCUSSION}

CDDP has been the key drug in the chemotherapy of HNSCC for many years. In 1962, it was shown to have a relatively wide antitumour spectrum in animals (Rosenberg et al, 1969; Cvitkovic et al, 1977), and clinical trials were started in 1972 under the guidance of the National Cancer Institute. However, because of the severe side-effects of kidney dysfunction, CDDP usage was stopped until massive water infusion and use of diuretics accompanying CDDP administration was shown to decrease CDDP's adverse effects (Blachley and Hill, 1981). Following this, CDDP was initially approved in Canada and America in 1978. Although it is a useful drug for head and neck cancer treatment, some tumours show CDDP resistance. In these cases, the effect of CDDP might be low but the adverse effects cannot be ignored.

In the treatment of other tumours, patient selection is performed using analysis of biomarker expression. Thus, HER2/ neu gene selection and expression of the HER2 protein determines whether breast cancer patients should receive trastuzumab treatment, saving $\$ 40000$ per patient annually. Similarly, for chronic marrow characteristic leukaemia, patient selection is achieved by inspection of the Bcr-Abl fusion protein in the imatinib mesylate, saving an estimated $\$ 80000$ per patient in medical expenses (Davis et al, 2009). Therefore, identifying a true CDDP-resistant factor(s) as an effective biomarker for use in CDDP treatment would contribute to the medical economy.

Several previous studies have investigated mechanisms of CDDP resistance, including decreasing the CDDP concentration inside the cell by increasing expression of the ATP-binding cassette transporter family and the excretory drug pump in the cell membrane (Taniguchi et al, 1996); increasing the expression of the detoxification proteins glutathione S-transferase- $\pi$ (GST- $\pi$ ) and metallothionein inside the cell (Chen et al, 1989; Nakano et al, 2003); and restoring expression of the excision repair crosscomplementing one mismatch repair enzyme (Fink et al, 1996). However, the true resistance factors remain elusive.

The difficulty in searching for CDDP-resistant elements might be because HNSCC is caused by several different factors such as 


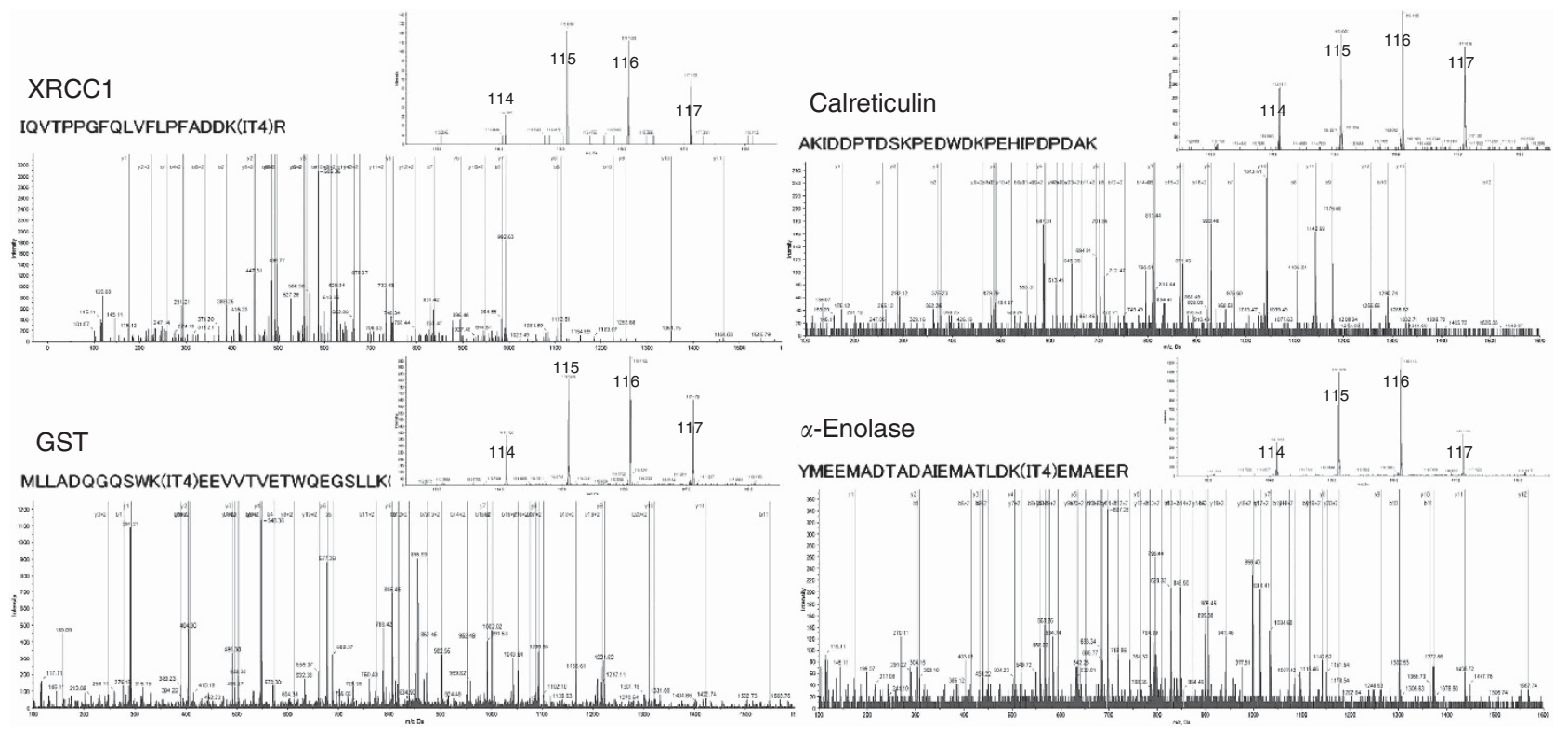

Figure 3. MS/MS spectra of select peptides with their reporter ions for the four proteins XRCC1, calreticulin, GST, and $\alpha$-enolase. Representative peptide sequencing and quantification using iTRAQ with indicated amino acid sequences, annotated b-ion and y-ion series, and an expanded view of the reporter ion region showing representative relative abundances of signature iTRAQ ions at $\mathrm{m} / \mathrm{z} 114,115,116$, and 117 .
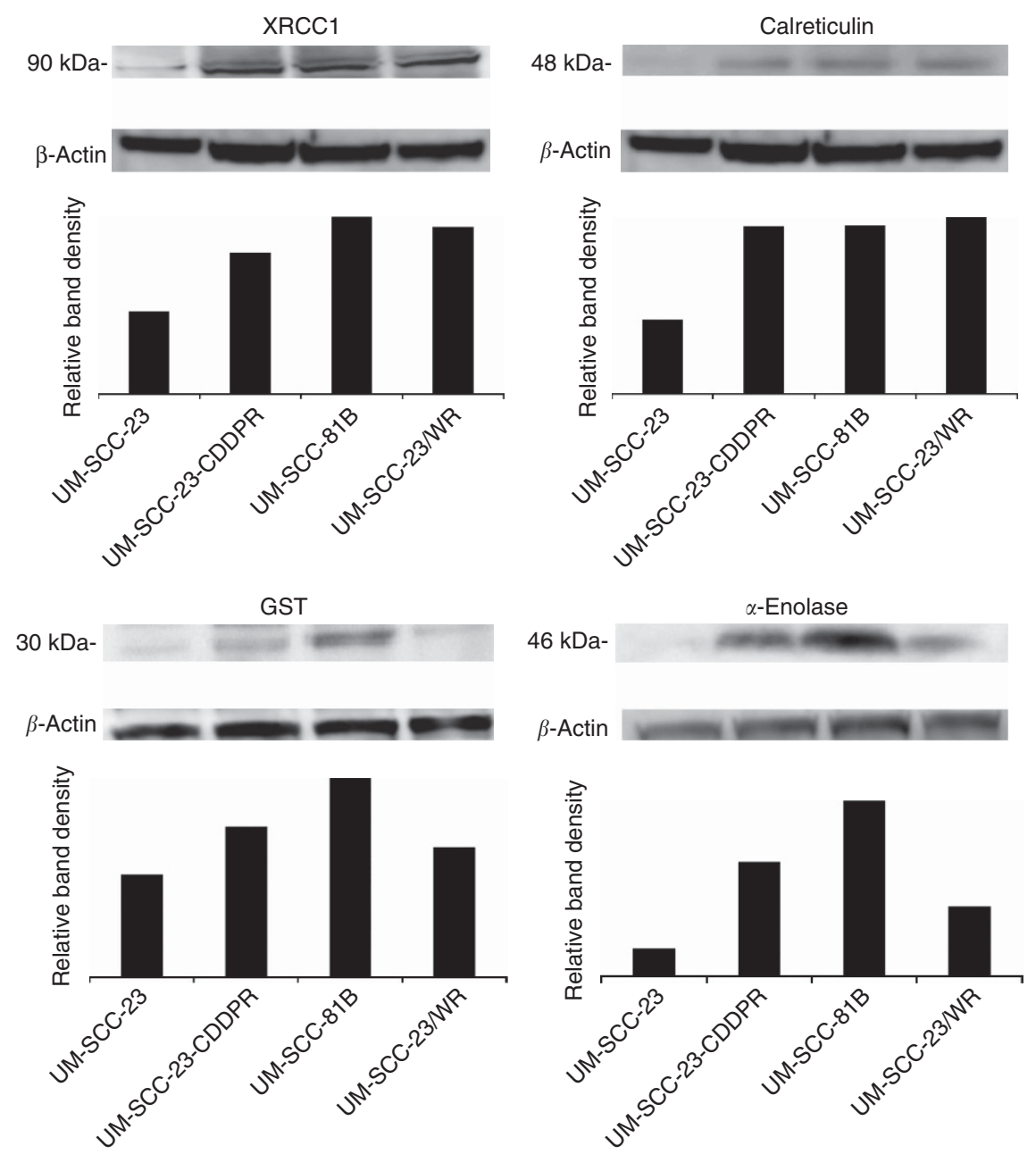

Figure 4. Validation of iTRAQ results by western blotting for XRCC1, calreticulin, GST, and $\alpha$-enolase. The revelation was checked for these proteins by western blotting, with the same result obtained as for ProteinPilot. The density of each band was measured and normalized against that of $\beta$-actin. Beta-actin was used as an internal loading control. 


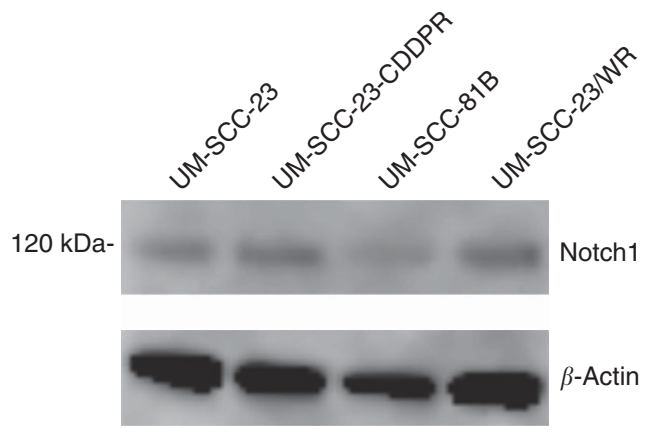

Figure 5. Expression of NOTCH1 by western blot analysis in each cell line. The expression of NOTCH1 was seen in all the cell lines but slightly decreased in UM-SCC-81B.

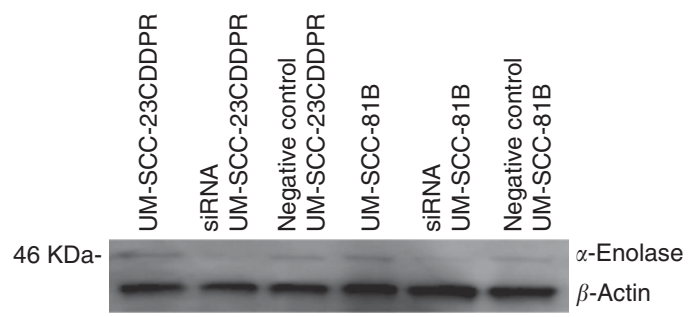

Figure 6. Alpha-enolase knockdown effect using siRNA was analyzed by western blot analysis in UM-SCC-23-CDDPR and UM-SCC-81B cell lines. High $\alpha$-enolase expression was detected in both cell lines. The effect of knockdown of $\alpha$-enolase was determined in UM-SCC-23CDDPR and UM-SCC-81B cells. The density of each band was measured and normalised against that of $\beta$-actin. Beta-actin was used as an internal loading control. As a result of rectifying by $\beta$-actin, this experiment is conducted correctly (Image Quant TL version 7.0 GE Healthcare, England).

smoking, drinking alcohol, and infection with viruses including human papillomavirus and Epstein-Barr virus (Liebowitz, 1994; Yuspa, 2000). The earlier studies might therefore have been hampered by the complex influences of these primary factors. In the present research, we used cell lines established from HNSCCs to decrease these influences. We compared protein expression between CDDP-sensitive and -resistant cell lines to identify a true resistance marker. We included the UM-SCC-81B cell line with natural resistance to CDDP to exclude the effect of repeated CDDP exposure on the resistance mechanisms. Also, to verify that the identified protein was specific for CDDP resistance rather than MDR, we used the 5-FU-resistant cell line UM-SCC-23/WR.

We employed the iTRAQ method to analyse protein expression levels combined with LC-ESI-MS/MS (Triple TOF5600). This differs from the high-throughput method that identifies and measures relative protein expression (Wang et al, 2011). We identified a total of 2173 proteins with differential expression between cell lines. Until now, it has not been possible to accurately detect more than 2000 proteins by single-cell analysis, but our method and the MS protocol enabled us to perform comprehensive analysis at the protein level and detect true chemoresistance factors. Our research previously identified 13 proteins, including XRCC1 and calreticulin, as potential MDR factors. Seven other proteins, such as GST and $\alpha$-enolase, were identified as being potentially specific for CDDP resistance. These proteins are reported to participate in chemoresistance and mediate cancer progression (Tew, 1994; Weaver et al, 2005; Tsaia et al, 2010; Sheng et al, 2013).

These findings suggest that in cases where MDR factors are detected, the clinician could select another treatment modality such as surgery. For tumours in which these proteins are highly expressed, avoiding anticancer drug administration is recommended as the effects would likely be limited. Thus, different treatment options should be considered, such as EGFR inhibitors or early surgical operation. Alternatively, when expression of these factors specific for CDDP resistance are detected, other anticancer drugs such as docetaxel or 5-FU could be indicated treatments. This strategy may be beneficial for the head and neck oncologist of our institution.

Functional analysis was conducted on $\alpha$-enolase in the seven identified CDDP-specific resistant proteins. The reason for selecting $\alpha$-enolase is that cancer cells have a high energy requirement for growth, and as $\alpha$-enolase is a glycolytic system component, it has an important role in cancer development (Jin et al, 2009). Therefore, we considered $\alpha$-enolase to have a specific role in the CDDP-specific resistant cell line.

Alpha-enolase is an enzyme that converts 2-phosphoglycerate and phosphoenolpyruvic acid in the glycolysis releasing system. Recent studies have shown that some glycolytic enzymes are complex, multifaceted proteins rather than simple components of the glycolytic pathway (Kim and Dnag, 2005; Tsaia et al, 2010). It is thought that the energy released by the glycolytic system is used not only for tumour growth, but also for tumour tolerance, such as discharge of the anticancer drugs or their metabolites from a cell. We, therefore, considered that $\alpha$-enolase has the potential to reinforce the cell's capability for DNA repair, as it was upregulated only in the CDDP-specific resistant cells. Moreover, Chang et al (2003) reported that $\alpha$-enolase has a latent role in tumour occurrence, as it appears to be an important factor in the high metabolism of cancer cells, and that its expression might be related to cancer infiltration and metastasis. Although detection of $\alpha$-enolase antigens in non-small cell lung cancer patients was shown to be associated with tumour recurrence (Chang et al, 2006), no studies have investigated its role in CDDP resistance. We, therefore, carried out functional analysis of $\alpha$-enolase using siRNA but found no significant effect of $\alpha$-enolase siRNA on UM-SCC-23/WR (5-FU-resistant) or UM-SCC-23-CDDPR (acquired CDDP-resistant) cells. On the other hand, CDDP sensitivity was increased significantly in UM-SCC-81B (naturally CDDP-resistant) cells after siRNA knockdown of $\alpha$-enolase.

On the basis of these findings, $\alpha$-enolase might be a true CDDP chemoresistance factor in natural cisplatin resistance. Furthermore, the findings of natural CDDP resistance and acquired CDDP resistance being different phenomena of the siRNA knockdown of $\alpha$-enolase suggest that the acquired resistance mechanisms for CDDP differ from the natural resistance mechanisms. Indeed the expression level of Notch1 on UM-SCC-23s are slightly higher than UM-SCC-81B. Notch pathway has a tumour suppressor role in head and neck squamous cell carcinoma (Pickering et al, 2013). Also there is a report related to Notch1 cooperation fact with $\alpha$-enolase. The Activated Notch1 receptor cooperates with $\alpha$-enolase and MBP-1 in modulating c-myc activity. (Hsu et al, 2008). Thus, future work should identify and compare these mechanisms as they might have a clinical bearing on head and neck cancer treatment. Alpha-enolase is a potential CDDP-specific resistant factor, and in the future it might serve as a useful biomarker of CDDP resistance in cancer therapy. Detection of $\alpha$-enolase could therefore allow clinicians to select appropriate anticancer agents in $\mathrm{HN}$ treatment. Additional research is expected to focus on these properties of $\alpha$-enolase on HNSCC.

It has long been anticipated that effective cancer treatment markers could be identified, and the recent progress in mass spectrometry has enabled us to perform comprehensive analysis at the protein level. To our knowledge, this is the first report of the use of the iTRAQ method and LC-ESI-MS/MS to investigate the anticancer drug resistance mechanisms of CDDP and 5-FU from the perspective of head and neck cancer treatment. Our results 

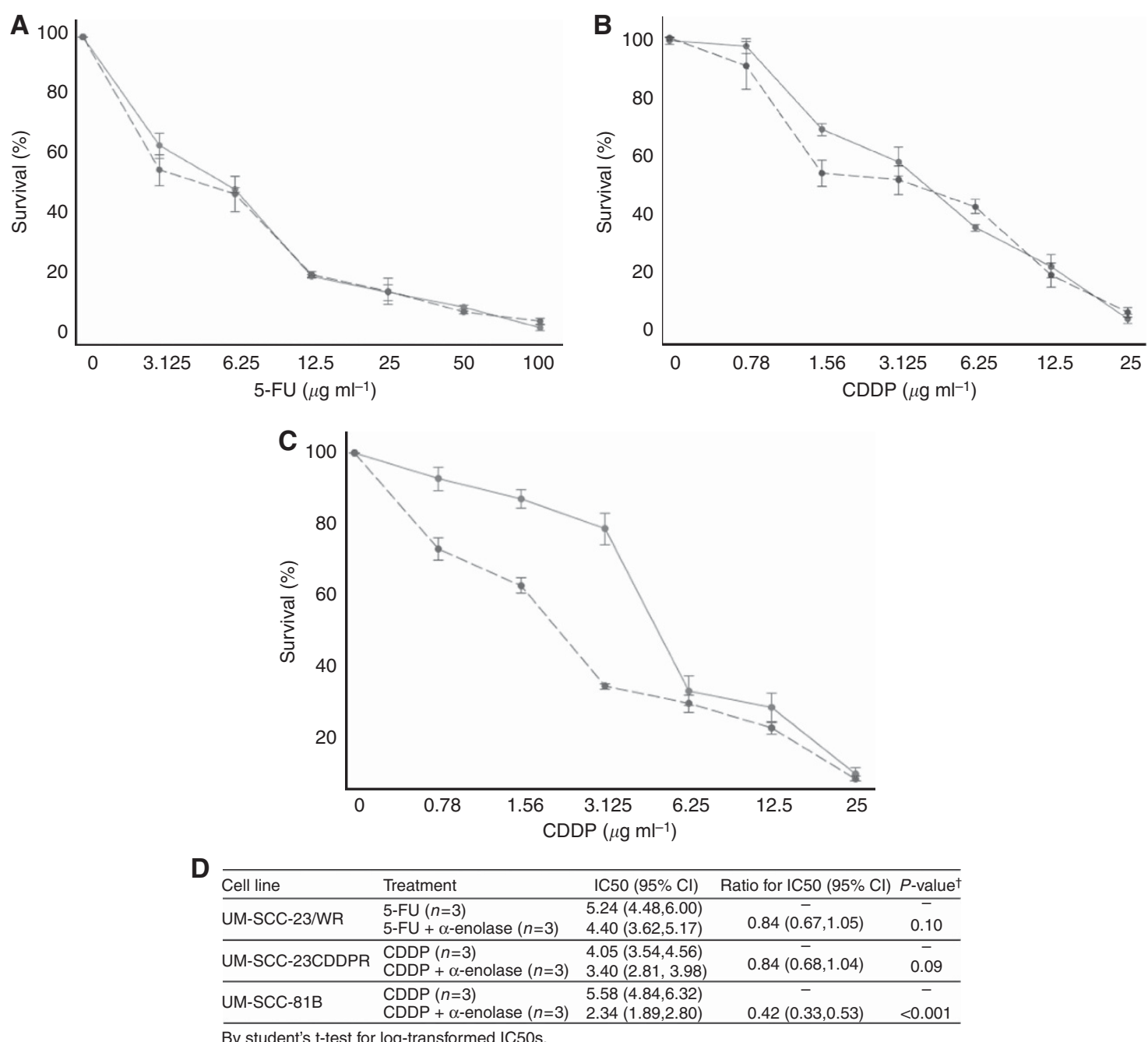

Figure 7. Changes to anticancer drug sensitivity after $\alpha$-enolase siRNA in each cell line. Bars represent the standard deviation. (A) UM-SCC-23/ $\mathrm{WR}+(5-\mathrm{FU})$ (solid line), UM-SCC-23/WR + (5-FU $+\alpha$-enolase siRNA) (dashed line). The effect of combination treatment using $\alpha$-enolase siRNA and 5-FU on the proliferation of UM-SCC-23/WR was examined by WST-1 assay. (B) UM-SCC-23CDDPR + (CDDP) (solid line), UM-SCC$23 C D D P R+(C D D P+\alpha$-enolase siRNA) (dashed line). The effect of combination treatment using $\alpha$-enolase siRNA UM-SCC-23CDDPR was examined by WST-1 assay. (C) UM-SCC-81B + (CDDP) (solid line), UM-SCC-81B + (CDDP $+\alpha$-enolase siRNA (dashed line). The effect of combination treatment using $\alpha$-enolase siRNA UM-SCC-81B was examined by WST-1 assay. (D) Comparison for IC50. ${ }^{\dagger}$, Student's t-test was used for comparison of log-transformed IC50 between with and without $\alpha$-enolase.

indicate that known resistant cases can be treated with decreased quantities of anticancer drugs, and that curative effects can be envisaged even for sensitive cases before clinical treatment. Also, by jointly using inhibitors of anticancer drugs that were discovered in our experiment, we can expect to offer new treatment strategies in the near future.

\section{ACKNOWLEDGEMENTS}

This work was supported by a grant from the Japan Society for the Promotion of Science and a Grant-in Aid for Science Research from the Ministry of Education, Culture, Sports, Science, and Technology, Japan.

\section{CONFLICT OF INTEREST}

The authors declare no conflict of interest.

\section{REFERENCES}

Blachley JD, Hill JB (1981) Renal and electrolyte disturbances associated with cisplatin. Ann Intern Med 95: 628-6632.

Boring CC, Squires TS, Tong T (1993) Cancer statistics 1993. CA Cancer J Clin 43: 7-26.

Brenner JC, Graham MP, Kumar B, Saunders LM, Kupfer RK, Lyons RH, Bradford CR, Carey TE (2010) Genotyping of 73 UM-SCC head and neck squamous cell carcinoma cell lines. Head Neck 32: 417-426.

Chang YS, Wu W, Walsh G, Hong WK, Mao L (2003) Enolase-alpha is frequently down-regulated in non-small cell lung cancer and predicts aggressive biological behavior. Clin Cancer Res 9: 3641-3644.

Chang GC, Liu KJ, Hsieh CL, Hu TS, Charoenfuprasert S, Liu HK, Luh KT, Hsu LH, Wu CW, Ting CC, Chen CY, Chen KC, Yang TY, Chou TY, Wang WH, Whang-Peng J, Shih NY (2006) Identification of alpha-enolase as an autoantigen in lung cancer: its overexpression is associated with clinical outcomes. Clin Cancer Res 12: 5746-5754.

Chen G, Frei E, Zeller WJ (1989) Determination of intracellular reduced glutathione and glutathione related enzyme activities in cisplatin-sensitive and resistant experimental ovarian carcinoma cell lines. Cancer Lett 46: 207-211. 
Chung CH, Parker JS, Karaca G, Wu J, Funkhouser WK, Moore D, Butterfoss D, Xiang D, Zanation A, Yin X, Shockley WW, Weissler MC, Dressler LG, Shores CG, Yarbrough WG, Perou CM (2004) Molecular classification of head and neck squamous cell carcinomas using patterns of gene expression. Cancer Cell 5: 489-500.

Chung CH, Levy S, Yarbrough WG (2006) Clinical applications of genomics in head and neck cancer. Head Neck 28: 360-368.

Chung CH, Levy S, Chaurand P, Carbone DP (2007) Genomics and proteomics: emerging technologies in clinical cancer research. Crit Rev Oncol Hematol 61: 1-25.

Craft GE, Chen A, Nairn AC (2013) Recent advances in quantitative neuroproteomics. Methods 61: 186-218.

Cvitkovic E, Spaulding J, Bethune V, Martin J, Whitmore WF (1977) Improvement of cis-dichlorodiammineplatinum (NSC 119875): therapeutic index in an animal model. Cancer 39: 1357-1361.

Davis JC, Furstenthal L, Desai AA, Norris T, Sutaria S, Fleming E, Ma P (2009) The microeconomics of personalized medicine: today's challenge and tomorrow's promise. Nat Rev Drug Discov 8: 279-286.

Fink D, Nebel S, Aebi S, Zheng H, Cenni B, Nehmé A, Christen RD, Howell SB (1996) The role of DNA mismatch repair in platinum drug resistance. Cancer Res 56: 4881-4886.

He H, Sun G, Ping F (2009) Laser-capture microdissection and protein extraction for protein fingerprint of OSCC and OLK. Artif Cells Blood Substit Immobil Biotechnol 37: 208-213.

Hsu KW, Hsieh RH, Lee YH, Chao CH, Wu KJ, Tseng MJ, Yeh TS (2008) The Activated Notch1 Receptor Cooperates with $\alpha$-Enolase and MBP-1 in Modulating c-myc Activity. Mol Cell Biol 15: 4829-4842.

Ijichi K, Adachi M, Hasegawa Y, Ogawa T, Nakamura H, Kudoh A, Yasui Y, Murakami S, Ishizaki K (2008) Pretreatment with 5-FU enhances cisplatin cytotoxicity in head and neck squamous cell carcinoma cells. Cancer Chemother Pharmacol 62: 745-752.

Jin S, DiPaola RS, Mathew R, White E (2009) Metabolic catastrophe as a means to cancer cell death. J Cell Sci 120: 379-383.

Kim JW, Dnag CV (2005) Multifaceted roles of glycolytic enzymes. Trends Biochem Sci 30: 142-150.

Liebowitz D (1994) Nasopharyngeal carcinoma: the Epstein-Barr virus association. Semin Oncol 21: 376-381.

Mignogna MD, Fedele S, Lo Russo L (2004) The World Cancer Report and the burden of oral cancer. Eur J Cancer Prev 13: 139-142.

Murata S, Adachi M, Kioi M, Torigoe S, Ijichi K, Hasegawa Y, Ogawa T, Bhayani MK, Lai SY, Mitsudo K, Tohnai I (2011) Etodolac improves 5-FU sensitivity of head and neck cancer cells through inhibition of thymidylate synthase. Anticancer Res 31: 2893-2898.
Nakano M, Sogawa CA, Sogawa N, Mishima K, Yamachika E, Mizukawa N, Fukunaga J, Kawamoto T, Sawaki K, Sugahara T, Furuta H (2003) Expression pattern of cisplatin-induced metallothionein isoforms in squamous cell carcinoma. Anticancer Res 23: 299-303.

Pickering CR, Zhang J, Yoo SY, Bengtsson L, Moorthy S, Neskey DM, Zhao M, Ortega Alves MV, Chang K, Drummond J, Cortez E, Xie TX, Zhang D, Chung W, Issa JP, Zweidler-McKay PA, Wu X, El-Naggar AK, Weinstein JN, Wang J, Muzny DM, Gibbs RA, Wheeler DA, Myers JN, Frederick MJ (2013) Integrative genomic characterization of oral squamous cell carcinoma identifies frequent somatic drivers. Cancer Discov 3(7): 770-781.

Rosenberg B, VanCamp L, Trosko JE, Mansour VH (1969) Platinum compounds: a new class of potent antitumour agents. Nature 222: 385-386.

Sheng W, Chen C, Dong M, Zhou J, Liu Q, Dong Q, Li F (2013) Overexpression of calreticulin contributes to the development and progression of pancreatic cancer. J Cell Physiol 229: 887-897.

Taniguchi K, Wada M, Kohno K, Nakamura T, Kawabe T, Kawakami M, Kagotani K, Okumura K, Akiyama S, Kuwano M (1996) A human canalicular multispecific organic anion transporter (cMOAT) gene is overexpressed in cisplatin-resistant human cancer cell lines with decreased drug accumulation. Cancer Res 56: 4124-4129.

Tew KD (1994) Glutathione-associated enzymes in anticancer drug resistance. Cancer Res 54: 4313-4320.

Tsaia ST, Chien IH, Shen WH, Kuo YZ, Jin YT, Wong TY, Hsiao JR, Wang HP, Shin NY, Wu LW (2010) ENO1, a potential prognostic head and neck cancer marker, promotes transformation partly via chemokine CCL20 induction. Eur J Cancer 46: 1712-1723.

Vokes EE, Weichselbaum RR, Lippman SM, Hong WK (1993) Head and neck cancer. N Engl J Med 328: 184-194.

Wang C, Guo K, Gao D, Kang X, Jiang K, Li Y, Sun L, Zhang S, Sun C, Liu X, Wu W, Yang P, Liu Y (2011) Identification of transaldolase as a novel serum biomarker for hepatocellular carcinoma metastasis using xenografted mouse model and clinic samples. Cancer Lett 313: 154-166.

Weaver DA, Crawford EL, Warner KA, Elkhairi F (2005) ABCC5, ERCC2, XPA and XRCC1 transcript abundance levels correlate with cisplatin chemoresistance in non-small cell lung cancer cell lines. Mol Cancer 4: 18.

Yuspa SH (2000) Overview of carcinogenesis: past, present and future. Carcinogenesis 21: 341-344.

This work is published under the standard license to publish agreement. After 12 months the work will become freely available and the license terms will switch to a Creative Commons AttributionNonCommercial-Share Alike 3.0 Unported License. 